\title{
Alphabet, Bibel, Katechismus: Das ABC der vormodernen Grundschule in den Niederlanden
}

\section{John Exalto}

\section{Zeitschrift für}

Erziehungswissenschaft

ISSN 1434-663X

Volume 15

Supplement 2

Z Erziehungswiss (2012) 15:65-77

DOI 10.1007/s11618-012-0311-y

\section{Stephanie Hellekamps \\ Jean-Luc Le Cam \\ Anne Conrad (Hrsg.) \\ Schulbücher \\ und Lektüren \\ in der vormodernen Unterrichtspraxis}

Zeitschrift für

ZfE Erziehungswissenschaft

\section{Springer VS}

Springer 
Your article is protected by copyright and all rights are held exclusively by Springer Fachmedien Wiesbaden. This e-offprint is for personal use only and shall not be selfarchived in electronic repositories. If you wish to self-archive your work, please use the accepted author's version for posting to your own website or your institution's repository. You may further deposit the accepted author's version on a funder's repository at a funder's request, provided it is not made publicly available until 12 months after publication. 


\title{
Alphabet, Bibel, Katechismus: Das ABC der vormodernen Grundschule in den Niederlanden
}

\section{$\boldsymbol{Z f E}$}

John Exalto

\begin{abstract}
Zusammenfassung: An Schulbüchern und Kinderliteratur der Frühmoderne ist in der modernen Pädagogik oft scharfe Kritik geübt worden, weil sie nur auf mechanisches Lernen gerichtet gewesen seien. In diesem Artikel wird behauptet, dass die gängige Auffassung diesem Unterrichtsmaterial nicht gerecht wird und dass es hingegen zur Bildung einer bürgerlichen Kultur mit einem allgemein-christlichen Gepräge beigetragen hat. Diese Hypothese wird beschrieben anhand einer Analyse des Schulprogramms der Grundschule in der niederländischen Republik. In diesem Schulprogramm waren Leseunterricht und Religionsunterricht aufs engste verbunden. Das Programm entsprach den Wünschen der reformierten Kirche, die ihre Mitglieder zu selbständigen Bibellesern ausbilden wollte. Das Abc-Buch wurde kalvinisiert, die Kinder lernten den Heidelberger Katechismus und Fortgeschrittenen wurde die sogenannte Testamentenliteratur angeboten. Diese kalvinistische Bildungsoffensive war nicht typisch niederländisch, sondern soll als eine Variante der europäischen Konfessionalisierung betrachtet werden.
\end{abstract}

Schlüsselwörter: Glaubensunterricht $\cdot$ Kalvinismus $\cdot$ ABC-Buch (Fibel) $\cdot$ Katechismus · Grundschule

\section{Alphabet, Bible, Catechism: The ABC of the early modern elementary school in the Netherlands}

\begin{abstract}
In modern pedagogy, schoolbooks and children's literature from the early modern period have encountered sharp criticism because they only focused on mechanical learning. This paper argues that this is an iniquity in respect to this schoolmaterial, but, by contrast, that these literature has contributed to the formation of a civic culture with a general Christian character. This hypothesis is described by an analysis of the school program of the elementary school in the Dutch Republic. In this program, reading education and religious education are inextricably linked. This way of teaching coincided with the goals of the Reformed Church to educate all its members to independent Bible readers. After the Reformation, the ABC Book was rewritten in a Calvinist way, children must learn the Heidelberg Catechism and to advanced students of the elementary school so-called testament literature was submitted. This Calvinist civilizing offensive was not typically Dutch, but must be considered as a variant of the European confessionalization in the early modern period.
\end{abstract}

(c) Springer Fachmedien Wiesbaden 2012

Dr. J. Exalto $(\square)$

Fakultät für Psychologie und Pädagogik, Erziehungsgeschichte,

Vrije Universiteit Amsterdam, Van der Boechorststraat 1, 1081 BT Amsterdam, Niederlande

E-Mail: j.exalto@vu.nl 
Keywords: Religious Education $\cdot$ Calvinism $\cdot$ ABC Book (Primer) $\cdot$ Catechism $\cdot$ Elementary School

Niederländische Schulbücher und Kinderlektüre aus der Periode vor 1800 haben nicht gerade einen guten Ruf. Das Niveau dieser Literaturgattung und mithin das Niveau der Grundschule sei hinter dem hohen Standard in den Bereichen der Kunst, Kultur und Wissenschaften, die in der frühen Neuzeit in den Niederlanden erblühten, zurückgeblieben. Auch der Leseunterricht sei unzureichend gewesen, da die Technik des Lesens ohne Rücksicht auf das Verständnis des Lesestoffs beigebracht worden sei. Auch wenn die Schüler die Technik beherrschten, sei das Textverständnis oft noch von nebensächlicher Bedeutung gewesen, was sich aus dem leiernden Ton, in dem gelesen wurde, ergebe. Auf diejenigen, die den mechanischen Lehrprozess durchgemacht hatten, habe eine große Enttäuschung gewartet: „Der farblose, geisttötende Lehrstoff muss für viele Kinder eine Plage gewesen sein“ (Bottema 1999, S. 55-57; vgl. Wouters und Visser 1926, S. 120; Boekholt und De Booy 1987, S. 80-85).

Diese Vorstellung findet sich häufig in der Historiographie und ist zum größten Teil auf die Aufklärungspädagogik zurückzuführen, in der scharfe Kritik an alten Unterrichtsmethoden geübt wurde und die eine große Transformation mit weitreichenden Folgen für die Gattung Schulbuch bewirkt hat. Die schlechte Reputation des niederländischen Schulbuches in der Periode vor 1700 hat dazu geführt, dass nur wenige Historiker diese Gattung erforscht haben. Dennoch scheint es mir zweckmäßig, den Schulbüchern, die in der Frühmoderne in der ,nederduitse' Schule, wie die Grundschule damals hieß, benutzt wurden, Aufmerksamkeit zu schenken. Meine - der gängigen Historiographie entgegengesetzte - Hypothese ist, dass die Schulbücher und die Kinderlektüre zur Bildung einer bürgerlich-christlichen, von biblischen Normen und Werten dominierten Kultur maßgeblich beigetragen haben.

Ich beschränke mich in diesem Beitrag auf den Grundschulunterricht in der niederländischen Republik der Frühmoderne, insbesondere im späten 16. und im 17. Jahrhundert, ungefähr also die Periode von der Reformation (1572) bis 1700. Mein Beitrag gliedert sich in vier Teile. Erstens behandle ich den geografischen, politischen und kulturellen Kontext der niederländischen Schule. Zweitens gehe ich auf die verschiedenen Schulbücher, die benutzt wurden, ein; dabei beschränke ich mich auf die bedeutendsten Schulfächer: Religion und Lesen. Drittens thematisiere ich das sogenannte Testament, eine Gattung für fortgeschrittene Schüler, die neues Licht auf den Charakter der kalvinistischen Bildungsoffensive mittels Schule und Lesekultur wirft. Zum Schluss gebe ich eine kurze Evaluation meiner oben erwähnten Hypothese.

Ich möchte in diesem Artikel das Unterrichtsprogramm beschreiben, das allen niederländischen Schülern angeboten wurde, wobei ich mich auf das Unterrichtsmaterial beschränke, das in den ersten drei Schuljahren eine Rolle spielte, weil eine relativ große Gruppe die Schule nach drei Jahren verließ. Dieses Unterrichtsprogramm wird als das „Abc“ der ,nederduitse“ Schule bezeichnet, weil Alphabet, Bibel und ,Catechismus“ im Mittelpunkt standen. Der Inhalt des Unterrichtsprogramms zeigt die enge Beziehung zwischen Religion und Unterricht. Im Abschnitt über das Testament überschreite ich die Grenzen des Abc, damit deutlich wird, wie das Abc weiter gestaltet wurde. 


\section{Alphabetisierung}

Die kalvinistische Reformation setzte sich in den Niederlanden nach 1580 durch und wurde zur öffentlichen Religion erhoben. Die reformierte Kirche wollte ihre Mitglieder zu selbständigen Bibellesern ausbilden und benutzte dazu den Grundschulunterricht. Die Grundschule wurde also zur Vorbereitung auf die Mitgliedschaft in der Kirche eingesetzt. Sowohl die Kirche als auch der Staat betrachteten die Schule als ein Instrument zur Disziplinierung der Gesellschaft. Die ,nederduitse“ Schule wurde kalvinisiert, aber ihre Zielsetzungen änderten sich nicht wesentlich. Genauso wie vor der Reformation bezweckte sie die Bildung guter Christen und tugendhafter Bürger. Der Religionsunterricht wurde selbstverständlich im reformatorischen Sinne geändert. Unter dem Einfluss der neuen reformierten Kirche wurde die Zahl der ,nederduitse' Schulen erweitert; ihr Bestreben, selbständige Bibelleser auszubilden, hat die Alphabetisierung in den Niederlanden stark angeregt. Die reformierte Kirche wurde aber nie Staatskirche und konnte ihren Willen deshalb den Bürgern nie aufzwingen. Der Staat erstellte die Schulordnungen. Die Reglements und ganz gewiss die Praxis gaben Andersdenkenden Raum. In Staats-Brabant, einer Provinz mit vielen katholischen Einwohnern, waren nur die reformierten Kinder verpflichtet, an dem Katechismusunterricht teilzunehmen. In den meisten Provinzen waren sogennante ,bijscholen' (Winkelschulen) neben den ,nederduitse' Schulen gestattet, Privateinrichtungen, die dankbar von den Katholiken benutzt wurden (vgl. Groenendijk 2007).

In der Förderung der Alphabetisierung war der Kalvinismus tonangebend. Aus lokalhistorischer Forschung ergibt sich, dass auch der katholische Klerus Alphabetisierung anstrebte, aber den Kalvinisten mit Abstand folgte (Roosenboom 1997). Die neuesten Erklärungen des hohen niederländischen Alphabetisierungsgrads weisen also nicht auf den Kalvinismus als einzigen Faktor hin. Vielmehr hatte die einzigartige Kombination von mehreren Faktoren zur Folge, dass die niederländische Republik im späten 16. und im 17. Jahrhundert den höchsten Alphabetisierungsgrad Europas erreichte. Die Grundlagen dafür waren bereits vor dem niederländischen Aufstand gegen Spanien und vor der Reformation gelegt worden. Im späten Mittelalter hatte sich in den Städten eine bürgerliche Kultur entwickelt, die in der föderativen, dezentralisierten Gesellschaft gedieh. Für die Teilnahme an der Diskussionskultur war Alphabetisierung erforderlich. Einen wichtigen Stimulus für die Lesekultur lieferte die Devotio Moderna.

Auch die wirtschaftliche Infrastruktur der bürgerlichen Gesellschaft, in der die Mittelschicht eine wichtige Rolle spielte, regte die Alphabetisierung an. Diese Entwicklung setzte ebenfalls bereits vor der Reformation mit der Einführung von kommerziellem Landbau und Viehzucht in den niederländischen Küstenprovinzen, dem spezialisierten Gewerbe und dem Ostseehandel ein. Im Goldenen Zeitalter entwickelte sich die Stadt Amsterdam zum wichtigsten europäischen Knotenpunkt des internationalen Handels. Sie verdankte ihre Position nicht zuletzt der günstigen geografischen Lage der niederländischen Republik an den Mündungen von Maas, Rhein und Schelde. Im Gefolge des internationalen Handels entwickelte sich auch ein lebendiger Kulturaustausch (vgl. Roding und Heerma van Voss 1996). Vor diesem ökonomischen Hintergrund fand im 17. Jahrhundert der Prozess der Verschriftlichung statt: Der Handel hatte seine Administration, die Kenntnisse im handwerklichen Sektor wurden mehr denn je in Handbüchern 
für Schüler schriftlich festgelegt, während das Angebot an populären Druckwerken zur politischen Meinungsbildung zunahm (Frijhoff und Spies 1999, S. 237-239; Otterspeer 2002; Frijhoff 2004). Im Laufe des 17. Jahrhunderts kam die sogenannte Französische Schule auf, eine weiterführende Ausbildung im Anschluss an die ,nederduitse' Schule. Neben Französisch, der Sprache der Handelskorrespondenz, wurde dort auch in Buchhaltung und Rechnen unterrichtet.

Die Entwicklungen in der niederländischen Republik waren sowohl Ursache als auch Folge des hohen Alphabetisierungsgrads. Verschiedene Prozesse beeinflussten sich gegenseitig. Es ist aber klar, dass es am Anfang dieser Kette eine Institution geben musste, in der das Alphabet gelernt wurde, und das war die ,nederduitse' Schule. Dort verbrachten die meisten niederländischen Kinder ab dem vierten oder fünften Lebensjahr drei Jahre. Weil Lesen und Schreiben strikt getrennt waren, ist der Alphabetisierungsgrad nicht anhand der Schreibfertigkeit zu bestimmen. Eine Berechnung ist daher nicht einfach. Es steht allerdings fest, dass eine Minorität der Gesellschaft, ungefähr zehn Prozent, gar nicht alphabetisiert war. Die Mehrheit war fähig, einfache Texte zu entziffern (vgl. für den Alphabetisierungsgrad: Van Deursen 1994, S. 131-143). Wir werden uns im Folgenden nicht mit dem weiteren Lebensgang des niederländischen Kindes beschäftigen, sondern beschränken uns auf den Lesestoff, der allgemeines Kulturgut war. Auf die Bücher also, die nahezu alle niederländischen Kinder im Leseunterricht benutzten.

\section{Erstleseunterricht}

Wer im Alter von vier oder fünf Jahren in die Schule ging, bekam Unterricht in Religion und Lesen. Die Fächer verstärkten sich gegenseitig: Kinder lernten das Lesen mithilfe religiöser Texte. Der Prozess des Lesenlernens dauerte etwa drei Jahre. Er folgte einer rigiden Systematik, nach der zuerst einzelne Buchstaben und Silben gelernt und dann Wörter und Sätze geübt wurden. Erst danach wurde das eigentliche Lesen gelernt. Der Lesestoff für Fortgeschrittene war nicht spezifisch für Kinder geeignet, was die Attraktivität erheblich geschmälert haben wird (De Booy 1977, S. 49-51; Boekholt und De Booy 1987, S. 37-38). Wenn Eltern nach dieser Lehrstufe noch Geld übrig hatten, konnten sie für ihre Kinder Schreibunterricht folgen lassen, doch das war eine Ausnahme. Eine noch kleinere Gruppe wurde auch in der Rechenkunst unterrichtet. Ich werde jetzt auf drei Kategorien von Unterrichtsmaterial eingehen. Erstens das Material für das Lesenlernen, zweitens das Material für den Religionsunterricht, und drittens die Lesebücher für jene Schüler, die das Buchstabieren schon beherrschten.

Das Unterrichtsmaterial für das Lesenlernen war schon jahrhundertelang das AbcBuch. Das niederländische Abc-Büchlein ist, wie die deutsche Fibel und das englische Primer, wegen seiner fragilen Ausführung und intensiven Benutzung nur sehr fragmentarisch überliefert. Der erste Leseunterricht wurde auch nach der Reformation mithilfe des Kleinen Abc-Buches und des Großen Abc-Buches erteilt. Der Inhalt wurde protestantisiert, denn neben dem Abc in Fraktur und italischer Schrift (Buchstaben, Vokale und Silben) gehörten das Pater noster, das Ave Maria und das Credo zum Inhalt des Großen Abc-Buches. Diese Texte waren ursprünglich in Latein und wurden durch protestantische Texte in der Volkssprache ersetzt. In der ältesten protestantischen Edition, die in den Nieder- 
landen verwendet wurde, findet man sechs sogenannte Kapitel der christlichen Lehre: das Vaterunser, das Apostolikum, die Zehn Gebote und einen kurzen Text über die heilige Taufe, einen über das heilige Abendmahl und einen über die christliche Strafe oder Zucht sowie einige Morgen- und Abendgebete.

Eingehende textkritische Forschung der überlieferten Fragmente hat ergeben, dass das niederländische Abc-Buch in London in der Periode 1550-1553 unter der Leitung von Jan Łaski (oder Johannes a Lasco, 1499-1560) verfasst wurde. Łaski war ein aus Polen gebürtiger Theologe, der die Leitung über die Flüchtlingsgemeinden hatte, wo zwischen 1540 und 1572 verfolgte reformierte Protestanten, die aus den Niederlanden vertrieben waren, eine Zuflucht fanden. In der bedeutendsten Flüchtlingsgemeinde, jener im ostfriesischen Emden, war Łaski mit seinen Mitarbeitern am Übergang zur Reformation beteiligt. Mit Jan Utenhove (1520-1565) und Marten Micron (1523-1559) leistete er einen wichtigen Beitrag zur Liturgie und Kirchenordnung. Emden war bis 1570 das Zentrum der protestantischen Buchdruckerkunst. Dort wurde z.B. in 1563 die Erstauflage der niederländischen Übersetzung des Heidelberger Katechismus gedruckt. Die niederländischen Flüchtlingsgemeinden gehörten zum europäischen Kräftefeld der religiösen Erneuerung; es gab einen lebhaften Austausch von Personen und Büchern mit anderen reformierten Zentren Europas, während Łaski auch mit seinem alten Lehrer Erasmus in Kontakt blieb. Offensichtlich fand man es auch wichtig, sich um die Jugend zu kümmern. Das niederländische Abc-Buch, das wegen des Hahnes auf dem Umschlag - ein internationaler Brauch, den Hahn findet man z. B. auch auf Lehrbüchern von Melanchthon - auch Hahnenbuch genannt wurde, war von den kirchlichen Leitern für den Zweck des Lesenlernens verfasst worden, bereitete die Kinder aber auch auf die verschiedenen Katechismen, die ebenfalls in Umlauf kamen, vor. Der offizielle Name des Hahnenbuches war Emder Bedingen, was ,Gebetsbüchlein aus Emden' bedeutet. Das Buch diente bis ungefähr 1800 als Modell für die niederländischen Abc-Bücher (Stellingwerff 1979; auf S. 63-77 ein Faksimile des einzig überlieferten, unvollständigen Drucks der Emder Bedingen; vgl. Stellingwerff 1994; siehe für Emden als Mutterkirche der niederländischen Protestanten, Pettegree 1992).

Der Inhalt des Hahnenbüchleins ist kennzeichnend für die enge Beziehung zwischen Lesen und Gottesdienst. In späteren Auflagen findet man oft noch ein „christliches Abc“ in Reimen mit moralisierenden Sprüchen (,Q. Quade Reden verderven goede Zeden“ - Böse Beispiele verderben gute Sitten) (Groot A/ B/ C/ Boek 1781). Mehrere Schulordnungen verordneten das Auswendiglernen der sechs Kapitel der christlichen Lehre. Auch die für die niederländische reformierte Kirche so wichtige Synode von Dordrecht (1618-1619) schrieb die Kapitel, denen vorzugsweise „eenighe voornaemste spreucken der h. Schrifture, tot Godsalicheyt verweckende“ (,einige wichtige Sprüche der heiligen Schrift, die zur Gottseligkeit erwecken“) angefügt werden sollten, den jüngsten Kindern vor (Van Veen und Groenendijk 1979, S. 12). Ab dem 18. Jahrhundert ist unter dem Einfluss der Aufklärungspädagogik scharfe Kritik an dem Auswendiglernen von Texten geübt worden. In diese Kritik wurde in der Historiographie oft eingestimmt, aber mittlerweile sind wir zur Erkenntnis gekommen, dass die frühmoderne Gesellschaft ganz anders funktionierte als die moderne Gesellschaft. Ein gut trainiertes Gedächtnis war damals sehr wichtig, und das Auswendiglernen hatte eine viel bedeutsamere Funktion als heute (Groenendijk 2001, S. 82). Es legte ein Fundament für das spätere Leben. Das endlose 
Buchstabieren des Vaterunsers muss oft mechanisch und nicht gerade ehrfürchtig gewesen sein, aber die Kirche scheint damit vorlieb genommen zu haben.

Das protestantische Hahnenbuch wurde nicht überall direkt nach 1572 eingeführt; an einigen Orten wurde das alte Unterrichtsmaterial erst ein halbes Jahrhundert später durch neues ersetzt, teils wegen der Anhänglichkeit von Eltern und Schulmeistern an die alten Texte, wie das Büchlein mit den sieben Bußpsalmen, und teils weil die Protestantisierung auf dem Lande manchmal nur langsam vorankam. Letztendlich aber siegte der Hahn aus Emden, und um 1640 bekam er Verstärkung, als eine Methode für fortgeschrittene Schüler, die das Abc-Buch durchgearbeitet hatten, eingeführt wurde (De Booy 1980, S. 42-43). Das Buch mit dem Titel Trap der jeugd (,Stufe der Jugend“) schloss an das Abc-Buch an und bot differenzierten Unterricht, indem jedes Kapitel einer höheren Stufe entsprach. Es wurden regionale Varianten herausgegeben, die die wichtigsten Ortsnamen der Provinz enthielten. Die meisten Stufen enthielten auch eine Liste biblischer Personen zum Auswendiglernen (De Booy 1980, S. 44; Boekholt und De Booy 1987, S. 38-40). Die enge Beziehung zwischen Lesen und Gottesdienst ergibt sich weiter aus dem religiösen Charakter der Trappen der jeugd und aus der Aufnahme eines kurzen Katechismus in einigen Editionen (Wouters und Visser 1926, S. 106; De Booy 1977, S. 44). Ebenso wie das Alphabet gehörte der Katechismus zum Grundprogramm der ,nederduitse“ Schule. Der Inhalt des Unterrichts der Grundschule ist als ,Alphabet, Bibel und Katechismus` definiert worden. Nach dem Alphabet behandeln wir jetzt die Bibel.

\section{Lektüren für fortgeschrittene Leser}

Das Hahnenbuch enthielt einige wichtige Kapitel der christlichen Lehre, die wörtlich aus dem Bibel entlehnt waren. Fortgeschrittenen Lesern wurde nach dem Hahnenbuch die Trap der jeugd angeboten und daneben gab es für sie sogenannte Lesebücher. Diese enthielten wörtliche Auszüge aus dem Alten Testament und waren manchmal mit Holzschnitten versehen. Einfache Lektüre war es nicht, aber es traf direkt die Zielsetzung der Kirche, durch den Unterricht selbständige Bibelleser auszubilden. Am meisten verbreitet seit 1572, seit der Reformation also, war De historie van den conincklycken propheet Davids („Die Historie des königlichen Propheten David“), deren Erstausgabe um 1600 publiziert wurde. An zweiter Stelle kamen die Proverbia ofte spreucken des alderwijsten koninghs Salomonis („Proverbia oder Sprüche des weisen Königs Salomo“), die ebenfalls um 1600 gedruckt wurden. Als 1637 die neue niederländische Bibelübersetzung erschien, die sogenannte ,Statenvertaling‘ oder Statenbibel, wurden die Lesebücher an diese Übersetzung angepasst.

Die Statenbibel hat einen wichtigen Beitrag zur Standardisierung der niederländischen Sprache geleistet; die Lesebücher, die auf der ,nederduitse ' Schule benutzt wurden, spielten darin eine wichtige Rolle. Der Theologe Willem van der Meiden hat darauf hingewiesen, dass die junge niederländische Republik zur Selbstbestätigung die alttestamentlichen Geschichten zum Vorbild nahm. Ihr Selbstverständnis als reformiertes Israel wurde am Alten Testament gespiegelt. Es gab mehrere protestantische Länder, die die Kirche metaphorisch als das protestantische Israel betrachteten (Ihalainen 2005). Obwohl die These Van der Meidens, die sich auf die Nation bezieht, anfechtbar ist, spricht viel für ein kirch- 
liches protestantisches Selbstverständnis in Analogie zu Israel, das den Bibelgeschichten umso mehr Kraft gab, auch wenn man sich fragen kann, ob die Kinder sich dieser Analogie bewusst waren (Van der Meiden 2009, S. 41-44).

Den Kindern wurden also wörtliche Bibeltexte angeboten. Gravurbibel, Frage- und Antwortbücher und Nacherzählungen von biblischen Geschichten datieren erst seit dem 18. Jahrhundert. Weil wir uns mit dem 16. und 17. Jahrhundert beschäftigen, lassen wir das beiseite (vgl. Van der Meiden 2009; Houtman 2010). Neben den Lesebüchern mit alttestamentlichen Bibelfragmenten wurde das sogenannte ,Evangelienbuch“ als Lesebuch benutzt. Das Evangelienbuch enthielt Auszüge aus den Evangelien, die im Laufe des kirchlichen Jahres im Gottesdienst gelesen wurden, die Haupttexte also für die christlichen Feste. Es war ein Erbe der katholischen Vergangenheit, das die reformierte Kirche wegen seiner Popularität nicht hatte ablehnen können. Der Text wurde selbstverständlich an die reformierte Liturgie und Bibelübersetzung angepasst. Bezüglich älterer Bücher mit Geschichten aus den Apokryphen, wie Tobias und Judith, machte die Reformation ihren Einfluss allerdings geltend. Diese Bücher wurden zwar nicht verboten, doch von ihnen wurde abgeraten, und als Ersatz erschienen die Bücher über David und die Sprüche (De Booy 1977, S. 42, 51-52, 269; De Booy 1980, S. 41-46; Bekkering 1990, S. 87, 113-115, 182; Van der Meiden 2009, S. 41, 44, 46-48).

Die dritte Komponente des ABC war der Katechismus. Die bereits erwähnten religiösen Lehrbücher standen immer im Dienst des Leseunterrichts - und ebenso gilt umgekehrt: Der Leseunterricht stand im Dienst der religiösen Bildung. Der Katechismus wurde auch für Buchstabier- und Leseübungen benutzt, namentlich während des besonders dafür reservierten Religionsunterrichts. Der Katechismus oder ein Auszug wurde dabei in der Klasse behandelt, während der Unterricht sonst individuell war. Die einfachen Fragen des Katechismus wurden vermutlich im Sprechchor beantwortet, wobei man unterschied zwischen den jüngsten Kindern, die nur die Fragen auswendig lernten, und den älteren Schülern, für die der Katechismusunterricht mit einer Auslegung durch den Schulmeister, Katechisiermeister oder Pfarrer verbunden war. Dieser Unterricht wurde an freien Mittwoch- oder Samstagmittagen erteilt, damit die katholischen Kinder ohne Probleme wegbleiben konnten, um ihren eigenen Glaubensunterricht an geheimen, aber tolerierten Orten zu genießen. Das war auf jeden Fall die Praxis in der Provinz StaatsBrabant, wo verhältnismäßig viele Katholiken wohnten (Roosenboom 1997, S.200). Der Religionsunterricht diente zur Vorbereitung auf den Sonntag: die Psalmen, die an der Reihe waren, wurden geübt - vermutlich wurde dem Schulmeister nachgesungen, weil die Kinder selbst nicht über einen Psalter verfügten - und der Katechismus, der im Gottesdienst behandelt werden sollte, wurde auswendig gelernt. Während des Gottesdienstes am Sonntagnachmittag, der immer dem Katechismus gewidmet war, durfte der beste Schüler der Klasse die Fragen des Heidelberger Katechismus, der behandelt wurde, beantworten.

Der Heidelberger Katechismus wurde auch ,Großer Katechismus' genannt, weil er eigentlich für Erwachsene bestimmt war. Die Kinder lernten ihn wahrscheinlich ohne nähere Auslegung auswendig, was dem Verständnis nicht förderlich gewesen sein wird, und benutzten ihn als Buchstabier- und Lesematerial. Diese Praxis war gegen den Willen der Kirche, die von Anfang an eine Differenzierung des Lehrstoffes bezweckte: ein Großer Katechismus für Erwachsene und für den dem Katechismus gewidmeten Gottesdienst, 
ein Kleiner Katechismus für Jugendliche, die Mitglied der Kirche werden wollten, und eine noch einfachere Version für Schulkinder (Boekholt und De Booy 1987, S. 34-35). Die Grundlagen der Differenzierung wurden in Emden und London gelegt. Łaski und seine Mitarbeiter ,established a carefully graded hierarchy of catechismal works to bring members into full participation in Church life“ (Pettegree 1992, S. 24). In Emden wurden ein Großer und ein Kleiner Katechismus verfasst, und Marten Micron verfasste die Korte ondersoeckinghe des gheloofs (,Kurze Untersuchung des Glaubens“), die als Vorbereitung auf das Glaubensbekenntnis diente. Es war tatsächlich ,,a carefully graded hierarchy“: Die kleinsten Kinder lernten mithilfe der Emder Bedingen lesen und wurden über den Kleinen und den Großen Katechismus zur Korte ondersoeckinghe geführt. Łaski gab der Schule und der Kirche eine wichtige Rolle im Glaubensunterricht. Alle Kinder in Emden sollten zweimal jährlich von der Kirche examiniert werden. Aber auch die Familie war nach Łaski wichtig für die Glaubenserziehung (Verboom 1986, S. 77-85).

Ein wesentlicher Teil der reformierten Buchproduktion in Emden, von Andrew Pettegree genau inventarisiert, bestand aus katechetischem Unterrichtsmaterial. Daraus ergibt sich, dass die Kirche großen Wert auf die Glaubenserziehung legte, und es bestätigt die zentrale Rolle der Gattung Katechismus in der Reformation - manche reden von einer Katechismusoffensive. Das Emdener Unterrichtsmaterial wurde 1572 in die Niederlande gebracht. Einige Dezennien später fand das Cort begrijp („Kurze Zusammenfassung“, 1599) von Philips von Marnix, Herr von Sint Aldegonde (1540-1598) viel Anklang. Der Heidelberger Katechismus fehlte dem Emdener Unterrichtsmaterial als Grundlage, und da der Katechismus auch das reformierte Lehrbuch der niederländischen Kirche wurde, verschwand das Emdener Material nach einiger Zeit aus dem Unterricht zugunsten des Kort begrip der christelijke religie („,Kurze Zusammenfassung der christlichen Religion“, 1611), eine Zusammenfassung des Heidelberger Katechismus durch den reformierten Pfarrer Herman Faukelius (ca. 1560-1625). Das Buch von Marnix, das ebenso wenig auf den Heidelberger Katechismus gegründet war, wurde 1659 durch ein Buch, das diesen als Ausgangspunkt hatte, ersetzt. Der Hersteller, der reformierte Pfarrer Jacobus Borstius (1612-1680), gab dem Buch einen sprechenden Titel: Kort begrijp der christelijcke leer („Kurze Zusammenfassung der christlichen Lehre“, Groenendijk 2001).

Die Synode von Dordrecht von 1618-1619 - die letzte nationale Synode, die der Staat erlaubt hatte - folgte der Emdener Kirche, indem sie ,a carefully graded hierarchy“ vorschrieb: die sechs Kapitel der christlichen Lehre für die Kleinen, gefolgt durch den Katechismusauszug des Faukelius und durch den Heidelberger Katechismus selbst für die Fortgeschrittenen. Vermutlich wurde die katechetische Hierarchie nicht überall und immer respektiert. In der Schule rezitierten die Kinder schon den Heidelberger Katechismus und die Pfarrer überließen den Katechismusunterricht, den sie für unter ihrer Würde achteten, oft den Schul- und Katechisiermeistern (Van Veen und Groenendijk 1979). Das wiederholte Rezitieren dieser ,korte begrippen“ (kurze Zusammenfassungen) sorgte dafür, dass wenn nicht der Inhalt, so doch der Wortlaut der christlichen Lehre in den Köpfen vieler Kinder einen Platz bekam. Besonders in der zweiten Hälfte des 17. und im 18. Jahrhundert erweiterte sich die Zahl der Katechismusbücher erheblich; sie entsprachen oft nicht ihrer Zielsetzung, den Lehrstoff zu vereinfachen. Ich signalisiere diese Entwicklung, ohne näher auf sie einzugehen. Meiner Ansicht nach können wir feststellen, dass die reformierte Kirche mit der ,nederduitse' Schule, die das reformierte Abc (Alphabet, Bibel 
und Katechismus) unterrichtete, zufrieden sein durfte. Das Auswendiglernen dominierte zwar oft die Erklärung, aber die Situation war nach der Meinung von Zeitgenossen nicht derart schlecht, dass Reform notwendig war.

Ich kenne nur ein Beispiel von protestierenden Kindern. 1595 erhob sich gegen Meister Leenaert van Assendelft (in Noord-Holland) die Klage, er würde beim Leseunterricht immer dieselben Bücher benutzen, sodass die Kinder eine Abneigung gegen das Lernen bekamen (Van Deursen 1992, S. 144). Die Kinder ließen sich also nicht alles gefallen. Ob die Jugend aus Assendelft übermäßig kritisch war, ist nicht bekannt. Das Beispiel weist darauf hin, dass die Schulmeister sich Mühe geben mussten, um die Kinder sinnvoll zu beschäftigen.

Wir haben oben die gängigsten und wichtigsten Lehrbücher des reformierten Abc der ,nederduitse' Schule in der Periode 1580-1700 und ihren Inhalt erwähnt. Wahrscheinlich verfügten die Kinder über ein eigenes Exemplar des Abc-Buches. Die bekannten Gemälde von Schulinterieurs des 17. Jahrhunderts wie De schoolmeester („Der Schulmeister“; 1662) von Adriaen van Ostade (1610-1685) und De dorpsschool („Die Dorfschule, ca. 1670-1672) von Jan Steen (1626-1679) zeigen Kinder, die mit dünnen Büchern, meist in Oktav-Format, beschäftigt sind. Das Abc-Buch umfasste immer ein im Oktav-Format gefalztes Druckblatt. Möglicherweise zirkulierten auch Katechismusbücher im selben Format: der Text des Katechismus passte nämlich auf ein Druckblatt, und wir wissen, dass der Katechismus als Übungsstoff benutzt wurde. Psalmbücher waren viel umfangreicher und folglich teuerer; die Psalmen wurden vermutlich über Nachsingen des Schulmeisters, ohne Papierhilfsmittel gelernt.

\section{Testamente}

Ich habe das Schulprogramm fast aller niederländischen Kinder beschrieben, und könnte jetzt einen Schlusspunkt setzen. Ich möchte aber gern einen Abstecher machen, und eine unbekannte Gattung näher beleuchten, nämlich die des Testamentes. Unterschiedliche Bücher mit dem Titel ,Testament‘ wurden inner- und außerhalb der Schule jugendlichen Lesern, die buchstabierendes Lesen beherrschten, angeboten. Testamente werden zu den ältesten Kinderbüchern gezählt. Die Gattung des Testamentes beleuchtet den Charakter der kalvinistischen Bildungsoffensive über Schule und Lesekultur. Die Erforschung der Testamente vermag daher Einsicht in das Streben der reformierten Volkserzieher am Ende des 16. und im 17. Jahrhundert zu vermitteln. Der freie Lesestoff ist als Spiegelbild des formalen Lehrstoffes zu betrachten. Eines dieser Testamente ist bis zur Mitte des 17. Jahrhunderts als Lehrbuch für Fortgeschrittene benutzt worden. Der Titel dieses Buches Den uuterste wille (,Der letzte Wille“, 1565) verrät, dass das Mittelalter gar nicht so weit weg war. Der Autor, Lowys Porquin (1511-1573), war ein italienischer katholischer Geschäftsmann, der sich schon vor dem Aufstand und der Reformation in den nördlichen Niederlanden niedergelassen hatte. Das Testament, zunächst für seine Kinder geschrieben, wurde sowohl in den katholischen südlichen als auch in den protestantischen nördlichen Niederlanden benutzt, wahrscheinlich in Ermangelung einer besseren Alternative. Kinderbücher waren in dieser Zeit Mangelware. Offensichtlich war ein ausgeprägt reformierter Charakter der Schulbücher nicht erforderlich. Den uuterste wille ist 
die allgemein-christliche Ermahnung eines Vaters, ein Testament, in dem er seine Kinder zu einem christlichen und tugendhaften Leben aufruft (Greilsammer 1989).

Ein anderes bekanntes Buch aus dieser Zeit war das Testament der mennonitischen Märtyrerin Soetgen van den Houte $(\dagger 1560)$, das sie vor ihrer Hinrichtung im Gefängnis für ihre Kinder schrieb und das postum weite Verbreitung fand. Mehr als Porquin ermahnte sie ihre Kinder, den schmalen Weg zu gehen und die Eitelkeit zu fliehen. Ab 1699 erschien das Testament unter dem Titel Uyterste wille van Soetgen van den Houte („Letzte Wille von Soetgen van den Houte“) mit Stichen des bekannten Amsterdamer Dichters und Radierers Jan Luyken (1649-1712). Das Testament wurde bis weit ins 18. Jahrhundert regelmäßig neu gedruckt. Obwohl klar ist, dass das Buch besonders für Kinder gemeint war, wissen wir nicht, ob es in der Schule gelesen wurde. Um 1565 erschien eine deutsche Übersetzung, in der der Autorenname eingedeutscht wurde als Soecken von Holtz. Im 18. Jahrhundert wurde das Buch in einem Band mit dem gleichartigen, aus dem Englischen übersetzten Werk der Autorin Elisabeth Jocelyn (1596-1622) herausgegeben (vgl. Bekkering 1990, S. 149-155).

Außer Testamenten von Erwachsenen gab es auch Testamente von Kindern in der Kinderliteratur des 17. Jahrhunderts. Im 18. Jahrhundert wurde eine Sammlung dieser Kindertestamente unter dem Titel Des Heeren lof, verkondigt uit den mond der kinderen („Das Lob des Herrn verkündigt aus dem Mund der Kinder“", 1723), angefertigt von dem reformierten Pfarrer Wilhelmus Eversdijk (1653-1729), als Preis auf der ,nederduitse' Schule vergeben (De Booy 1977, S.272). Die Sammlung von Eversdijk ging über eine Übersetzung seines Kollegen Jacobus Koelman (1631-1695) von 1679 auf den in ganz Europa bekannten Sammelband A Token for Children (1671-1672) des englischen puritanischen Pfarrers James Janeway (1636-1674) zurück (vgl. Moore 2000; Groenendijk et al. 2010). Die Kindertestamente werden auch spirituelle Kinder(auto)biografien genannt. Die älteste niederländische reformierte Variante ist das Testament von Abigael Gerbrandts (ca. 1581-1600). In ihrem Sterbejahr erschien ein Buch unter dem Titel Een schoon testament ofte bekentenisse van een jonghe dochter (,Ein schönes Testament oder Bekenntnis einer jungen Tochter"), das in den ersten zwei Dezennien des 17. Jahrhunderts oft neu aufgelegt worden sein muss (Gerbrandts 1609). In diesem Buch beschreibt der Vater Abigaels das Sterben seiner Tochter und ihren gottesfürchtigen Lebenswandel. Die Geschichte ist ein Modell, ein Exempel. Zugleich kann man aus ihr erschließen, wie der Religionsunterricht gestaltet wurde.

Das Testament erzählt, dass Abigael in den Kinderjahren gern zur Schule ging. Mit siebzehn Jahren bekannte sie ihren Glauben. Zum Erstaunen der Presbyter konnte sie alle Fragen aus der Korte ondersoeckinghe des gheloofs von Micron fehlerfrei aufsagen. Sie ging gerne zur Kirche und hörte gerne Predigten. Ihrem Bruder Johannes gab sie Geld, damit er ihr aus der Bibel vorlas, während sie arbeitete. Selber las sie gern die Bibel ihres Großvaters, das Huysboec (1563) des schweizerischen Reformators Bullinger (15041575), die niederländische Übersetzung von dessen Hausbuch (1558; 1552 in Lateinisch erschienen), und De geschiedenisse ende den doodt der vromer martelaren (1559), das reformierte Buch der Märtyer des Pfarrers Adriaan van Haemstede (ca. 1525-1562). Aus dieser Geschichte ergibt sich, dass die Reformation schon ziemlich früh Erfolg hatte mit der Bildung selbständiger Bibelleser. Die Alphabetisierung ging sogar schneller voran als die Bibelverbreitung, denn Abigael las die Bibel ihres Großvaters. In ihrem Testa- 
ment warnte Abigael ihre Freundinnen vor weltlichem Aufwand; ihre Leser wurden angespornt, fromm und tugendhaft zu sein, die Bibel zu lesen, zur Kirche zu gehen und treu zu arbeiten. Wie die Testamente von Soetgen van den Houte und Elisabeth Jocelyn gehörte das der Abigael Gerbrandts zum kollektiven europäischen protestantischen Kulturgut der Frühmoderne. Es wurde 1650 in Sedan unter dem Titel Le testament ou confession de foy d'Abigail Gerbrants herausgegeben (Desgraves 1984-1985, Bd.2, S. 161, Nr. 4903).

\section{Fazit}

Tausende von Kindern haben die Abc-Bücher, die Bücher mit alttestamentlichen Bibeltexten, die kurzen Zusammenfassungen, die Katechismen und die Testamente inner- und außerhalb der Schule gelesen, dennoch wissen wir nur wenig über ihre Rezeption. Schulmeister Leenaert konnte sein Bücherangebot nicht ohne Folgen unverändert lassen. In diesem Beispiel hören wir indirekt die Stimme des Kindes, das sich nach etwas Neuem sehnte. Das Beispiel Abigaels zeigt, dass der Unterricht sich im reformatorischen Sinne änderte. Das Abc der ,nederduitse' Schule legte in erster Linie das Fundament für die Alphabetisierung, für das Lesenlernen, und danach bot es Zugang zur Bibel, zum Katechismus, zum reformierten Gut des Glaubens. Und schließlich gab es Testamente, die den Lesern ein tugendhaftes und gottesfürchtiges Leben und Aussicht auf die Ewigkeit vorhielten.

Dies alles war nicht typisch niederländisch. Ein polnischer Bischof, tätig in einer ostfriesischen Stadt und einige Zeit in Londen im Exil, legte das Fundament für das niederländische protestantische Abc. Ein italienischer katholischer Kaufmann erteilte eine Lektion in Rechtschaffenheit. Der Heidelberger Katechismus stammte aus dem Nachbarland, das Testament von Joycelin aus England. Zugleich wurde Soetgen ins Deutsche und Abigael ins Französische übersetzt. Die Benutzung vieler ausländischer Einflüsse und deren erfolgreiche Verarbeitung ist vielleicht charakteristisch für die Niederlande.

Die Schulbücher und die Kinderliteratur haben einen Beitrag zur Bildung einer bürgerlichen und allgemein-christlichen Kultur, in der biblische Normen für Lehre und Leben maßgeblich waren, geleistet. Deshalb verdient das Abc der ,nederduitse' Schule eine Rehabilitation. Zur weiteren Nuancierung des historiographischen Bildes ist nähere lokal-historische Erforschung des Funktionierens der ,nederduitse“ Schule sowie buchhistorische Erforschung der Drucke und Texte der Abc's, Katechismen und Testamente erforderlich. 


\section{Literatur}

\section{Quellen}

Gerbrandts, A. (1609). Een schoon testament/ ofte bekentenisse/ van een jonghe dochter gheheeten Abigail Garbrants/ oudt achthien jaren/ de welcke in een groote kranckheydt legghende aent roode melisoen/dese naebeschreven woorden op haar doot-bedde heeft ghesproken/ ende alsoo haer speel-ghenoodts ende jonghe maeghden tot Naerden/ oock andere die den Heere vreesen/tot leeringhe ende troost/ ter ghedachtenisse naegelaten. Met noch een beschrijvinghe van haer kindtsche dagen/ hoe datse haer tegen hare ouderen ghedraghen/ ende een leerkindt inde schole Jesu Christi/vander jonckheydt aen geweest is. Amsterdam: Hendrick Barentsz. [Staatsbibliothek Berlin, 4 an Dk 2880: S 16].

Groot A/ B/ C/ Boek. (1781) Zeer bekwaam voor de jonge kinderen te leeren. Amsterdam: Adam Meyer.

[Emder Bedingen] (1560-62) in J. Stellingwerff (1979). Kleine geschiedenis van het Groot abcboek of Haneboek. (S.63-77). 's-Gravenhage: Staatsdrukkerij.

\section{Sekundärliteratur}

Bakker, N., Noordman, J., \& Rietveld-van Wingerden, M. (2010). Vijf eeuwen opvoeden in Nederland. Idee en praktijk, 1500-2000 (2.Aufl.). Assen: Van Gorcum.

Bekkering, H. (Hrsg.). (1990). De hele Bibelebontse berg. De geschiedenis van het kinderboek in Nederland \& Vlaanderen van de middeleeuwen tot heden. Amsterdam: Querido.

Boekholt, P. T. F. M., \& Booy, E. P. de. (1987). Geschiedenis van de school in Nederland vanaf de middeleeuwen tot aan de huidige tijd. Assen: Van Gorcum.

Booy, E. P. de. (1977). De weldaet der scholen. Het plattelandsonderwijs in de provincie Utrecht van 1580 tot het begin der 19de eeuw. Utrecht: Stichtse Historische Reeks.

Booy, E. P. de. (1980). Kweekhoven der wijsheid. Basis- en vervolgonderwijs in de steden van de provincie Utrecht van 1580 tot het begin der 19e eeuw. Utrecht: Stichtse Historische Reeks.

Bottema, J. (1999). Naar school in de Ommelanden. Scholen, schoolmeesters en hun onderwijs in de Groninger Ommelanden, ca. 1500-1795. Bedum: Egbert Forsten \& Profiel.

Desgraves, L. (1984-1985). Répertoire des ouvrages de controverse entre Catholiques et Protestants en France (1598-1685) (2 Bde.). Genève: Librairie Droz S.A.

Deursen, A. T. van. (1992). Mensen van klein vermogen. Het kopergeld van de Gouden Eeuw. Amsterdam: Bakker.

Deursen, A. T. van. (1994). Een dorp in de polder. Graft in de zeventiende eeuw. Amsterdam: Bert Bakker.

Frijhoff, W. (2004). Calvinism, literacy, and reading culture in the early modern Northern Netherlands: Towards a reassessment. Archiv für Reformationsgeschichte, 95, 252-265.

Frijhoff, W., \& Spies, M. (1999). 1650. Bevochten eendracht. Den Haag: Sdu Uitgevers.

Greilsammer, M. (1989). Een pand voor het paradijs. Leven en zelfbeeld van Lowys Porquin, Piëmontees zakenman in de zestiende-eeuwse Nederlanden. Tielt: Lannoo.

Groenendijk, L. F. (2001). Marnix' kindercatechismus. In H. Duits \& T. van Strien (Hrsg.), Een intellectuele activist. Studies over leven en werk van Philips van Marnix van Sint Aldegonde (S. 76-86). Hilversum: Verloren. 
Groenendijk, L. F. (2007). Die reformierte Kirche und die Schule in den Niederlanden während des 16. und 17. Jahrhunderts. In H. Schilling \& S. Ehrenpreis (Hrsg.), Frühneuzeitliche Bildungsgeschichte der Reformierten in konfessionsvergleichender Perspektive. Schulwesen, Lesekultur und Wissenschaft (S. 47-74). Berlin: Duncker \& Humblot.

Groenendijk, L. F., Lieburg, F. A. van, \& Exalto, J. (2010). „Away with all my pleasant things in the world...": Model death-bed accounts of two young victims of the plague of 1664 in the Dutch town of Leyden. Paedagogica Historica, 46(3), 271-288.

Houtman, C. (2010). Bijbelse geschiedenis herverteld. Woord en beeld-vraag en antwoord. Heerenveen: Groen.

Ihalainen, P. (2005). Protestant nations redefined. Changing perceptions of national identity in the rhetoric of the English, Dutch and Swedish public churches, 1685-1772. Leiden: Brill.

Meiden, W. Van Der. (2009). Zoo heerlijk eenvoudig. Geschiedenis van de kinderbijbel in Nederland. Hilversum: Verloren.

Moore, C. N. (2000). „Gottseliges Bezeugen und frommer Lebenswandel.“ Das Exempelbuch als pietistische Kinderlektüre. In J. N. Neumann \& U. Sträter (Hrsg.), Das Kind in Pietismus und Aufklärung (S. 131-142). Tübingen: Max Niemeyer.

Otterspeer, W. (2002). Discipline en daadkracht. Onderwijs en wetenschap in Holland. In T. de Nijs \& E. Beukers (Hrsg.), Geschiedenis van Holland, Bd. 2: 1572 tot 1795 (S.331-352). Hilversum: Verloren.

Pettegree, A. (1992). Emden and the Dutch Revolt. Exile and the development of Reformed protestantism. Oxford: Clarendon.

Roding, J., \& Heerma Voss, L. van. (Hrsg.). (1996). The North Sea and culture (1550-1800). Hilversum: Verloren.

Roosenboom, H. T. M. (1997). De dorpsschool in de Meijerij van 's-Hertogenbosch van 1648 tot 1795. Tilburg: Stichting Zuidelijk Historisch Contact.

Stellingwerff, J. (1979). Kleine geschiedenis van het Groot abc-boek of Haneboek. 's-Gravenhage: Staatsdrukkerij.

Stellingwerff, J. (1994). Het abc der Gereformeerde Reformatie in de Nederlanden. Transparant, $5(4), 4-8$.

Veen, S. D. van, \& Groenendijk L. F. von. (Hrsg.). (1979). Het godsdienstonderwijs en de aanneming tot lidmaten in de gereformeerde kerk (2.Aufl.). Dordrecht: van den Tol.

Verboom, W. (1986). De catechese van de Reformatie en de Nadere Reformatie. Amsterdam: Buijten \& Schipperheijn.

Wouters, D., \& Visser, W. J. (1926). Geschiedenis van de opvoeding en het onderwijs, vooral in Nederland. Groningen: Noordhoff. 